\title{
The suppressor of cytokine signaling-1 (SOCS1) is a novel therapeutic target for enterovirus-induced cardiac injury
}

\author{
Hideo Yasukawa, ,2 Toshitaka Yajima, ${ }^{1,2}$ Hervé Duplain, ${ }^{1,2}$ Mitsuo Iwatate,, ${ }^{1,2}$ \\ Masakuni Kido, ${ }^{3}$ Masahiko Hoshijima, ${ }^{1,2}$ Matthew D. Weitzman, ${ }^{4}$ \\ Tomoyuki Nakamura,, ${ }^{1,2}$ Sarah Woodard, ${ }^{1,2}$ Dingding Xiong, ${ }^{1,2}$ Akihiko Yoshimura, ${ }^{5}$ \\ Kenneth R. Chien, ${ }^{1,2}$ and Kirk U. Knowlton ${ }^{1,2}$ \\ ${ }^{1}$ Institute of Molecular Medicine, \\ ${ }^{2}$ Department of Medicine, and \\ ${ }^{3}$ Division of Cardiothoracic Surgery, University of California at San Diego, La Jolla, California, USA \\ ${ }^{4}$ Salk Institute, San Diego, California, USA \\ ${ }^{5}$ Medical Institute of Bioregulation, Division of Molecular and Cellular Immunology, Kyushu University, Fukuoka, Japan
}

Enteroviral infections of the heart are among the most commonly identified causes of acute myocarditis in children and adults and have been implicated in dilated cardiomyopathy. Although there is considerable information regarding the cellular immune response in myocarditis, little is known about innate signaling mechanisms within the infected cardiac myocyte that contribute to the host defense against viral infection. Here we show the essential role of Janus kinase (JAK) signaling in cardiac myocyte antiviral defense and a negative role of an intrinsic JAK inhibitor, the suppressor of cytokine signaling (SOCS), in the early disease process. Cardiac myocyte-specific transgenic expression of SOCS1 inhibited enterovirus-induced signaling of JAK and the signal transducers and activators of transcription (STAT), with accompanying increases in viral replication, cardiomyopathy, and mortality in coxsackievirus-infected mice. Furthermore, the inhibition of SOCS in the cardiac myocyte through adeno-associated virus-mediated (AAV-mediated) expression of a dominant-negative SOCS1 increased the myocyte resistance to the acute cardiac injury caused by enteroviral infection. These results indicate that strategies directed at inhibition of SOCS in the heart and perhaps other organs can augment the host-cell antiviral system, thus preventing viral-mediated endorgan damage during the early stages of infection.

J. Clin. Invest. 111:469-478 (2003). doi:10.1172/JCI200316491.

\section{Introduction}

Enteroviral infection is a common cause of acute myocarditis that can lead to heart failure, arrhythmias, and death, especially among young adults and infants. In addition, enteroviral infection has been implicated in the development of dilated cardiomyopathy, one of the main indications for cardiac transplantation (1-3).

Received for publication July 24, 2002, and accepted in revised form January 5, 2003.

Address correspondence to: Kirk U. Knowlton, Department of Medicine and Institute of Molecular Medicine, University of California at San Diego, 9500 Gilman Drive, La Jolla, California 92093-0613K, USA. Phone: (858) 822-1364; Fax: (858) 822-3027; E-mail:kknowlton@ucsd.edu.

Hideo Yasukawa and Toshitaka Yajima contributed equally to this work.

Conflict of interest: The authors have declared that no conflict of interest exists.

Nonstandard abbreviations used: Janus kinase (JAK); suppressor of cytokine signaling (SOCS); adeno-associated virus (AAV); signal transducers and activators of transcription (STAT); cardiotrophin 1 (CT-1); leukemia inhibitory factor (LIF); coxsackievirus B3 (CVB3); dominant-negative SOCS (dnSOCS); left ventricular end-systolic dimension (LVESD); left ventricular end-diastolic dimension (LVEDD); percent fractional shortening (\%FS); IFN regulatory factor-1 (IRF1); $\alpha$-myosin heavy chain $(\alpha-\mathrm{MHC})$.
Both a direct viral cytopathic effect (4) and activation of the host cellular immune response $(1,5)$ play an important role in enterovirus-mediated myocarditis. Although there is considerable data regarding the role of the cellular immune response in viral myocarditis, little is known about the innate signaling mechanisms within the infected cardiac myocyte, their role in host-cell antiviral defense, and their contribution to susceptibility to myocarditis. In addition, there are no effective treatments that will inhibit replication of the virus in myocardium, especially in the early phase of viral infection (6).

IFNs are cytokines that play a central role in host defense against invasive viruses $(7,8)$. Elucidation of IFN signaling mechanisms led to the discovery of the Janus kinase (JAK) and the signal transducers and activators of transcription (STAT) signaling pathway that is required for expression of IFN-responsive genes (9-12). JAK-STAT activation results in induction of the suppressor of cytokine signaling (SOCS) family (12-17). Among the members of this family, SOCS1 and SOCS3 negatively regulate the JAK-STAT pathway by inhibiting JAK activity and thus inhibiting cytokine activity $(18,19)$. Cardiotrophin 1 (CT-1), leukemia inhibitory factor (LIF), and IL-6 also activate JAK-STAT signaling through gp130, a well-known cell-survival 
pathway in the cardiac myocyte that is negatively regulated by SOCS1 and SOCS3 $(20,21)$. The balance of this JAK-STAT-SOCS circuit determines the overall effect of cytokine stimulation (20).

It has been shown that administration of IFN- $\alpha$ or $-\beta$ can have a beneficial effect on viral myocarditis in the early stages of infection (22), but whole-animal knockouts of the IFN- $\alpha / \beta$ receptor had no detectable effect on the extent of viral infection in the heart during the early stages of infection in spite of a marked effect on viral replication in the liver (23). Furthermore, little is known regarding the effect of JAK-STAT activation by other cytokines, such as CT- 1 and IL-6, in viral heart disease. Therefore, the role for induction of the JAK-STAT signaling cascade within the infected cardiac myocyte is not clear.

We therefore set out to test the hypothesis that activation of JAK-STAT signaling within the cardiac myocyte is important for antiviral defense and that SOCS expression in the myocyte has a detrimental effect on the antiviral effect of JAK-STAT activation. Accordingly, in this study, we demonstrated that activation of the JAK-STAT pathway in the cardiac myocyte is upregulated and is required for efficient defense against the enterovirus-induced myocarditis, that cardiac-specific expression of SOCS1 has a detrimental effect on the development of virus-mediated heart disease, and that expression of a dominant-negative SOCS (dnSOCS) protein inhibits the virus-mediated myocytopathic effect.

\section{Methods}

Viruses. The coxsackievirus B3 (CVB3) used in this study was derived from the infectious CDNA copy of the cardiotropic H3 strain (Woodruff variant) of CVB3 (24). Virus titers were determined on HeLa cell monolayers using a standard plaque-forming assay, and virus isolation from heart and liver was performed as described previously (25). Recombinant adenovirus vectors containing the genes for LacZ, Myc-tagged SOCS1, Myc-tagged SOCS3, and Cre recombinase were prepared on 293 cells as described previously (26). Recombinant adenoviruses expressing dnSOCS1 driven by a CMV promoter were generated as described previously (27).

To generate adeno-associated virus (AAV) vectors containing dnSOCS1, the cDNA of Myc-tagged dnSOCS1 was subcloned into the AAV plasmid, pSub201 (28), under the control of a CMV immediateearly promoter site between the AAV inverted terminal repeats. Recombinant AAV-dnSOCS1 virus was generated by a triple-transfection protocol and purified as previously described (29).

Antibodies. Western blot and immunofluorescence staining were performed as described previously $(4,18)$ with the use of anti-STAT1, anti-phospho-STAT1, anti-STAT3, and anti-phospho-STAT3 antibodies (New England BioLabs Inc., Beverly, Massachusetts, USA); rabbit polyclonal anti-CVB3 antibody (a gift from A. Henke, Institute of Virology, Friedrich Schiller
University, Jena, Germany) (30); anti-c-Myc (A-14) antibody (Santa Cruz Biotechnology Inc., Santa Cruz, California, USA); and anti- $\beta$-galactosidase antibody (Cortex Biochem Inc., San Leandro, California, USA).

Generation of SOCS1 transgenic mice. The pGL- $\alpha \mathrm{MHC}-$ SOCS1 vector was constructed by subcloning the Myctagged SOCS1 cDNA (18) into the HindIII cloning site of the pGL- $\alpha M H C$ plasmid (generously provided by $T$. Kubota and J. Robbins). After digestion with BamHI, the fragment carrying the $\alpha \mathrm{MHC}$ promoter and SOCS1 cDNA was used for microinjection into (C57BL/6 X C3H) F1 (B6C3-F1) zygotes. Eggs surviving microinjection were transferred into the oviducts of recipient pseudopregnant females. Transgenic mice were identified by PCR analysis of tail genomic DNA, and protein expression was confirmed by immunoblotting with anti-Myc antibody (Santa Cruz Biotechnology Inc.). Four independent lines of transgenic mice were established. To perform experiments in an inbred line of SOCS1 transgenic mice that are susceptible to viral infection, we backcrossed three transgenic lines with $\mathrm{Balb} / \mathrm{c}$ mice for five generations.

Echocardiogram. Avertin (2.5\%) was administered intraperitoneally at $0.015 \mathrm{ml} / \mathrm{g}$ of body weight. Supplemental doses of anesthesia were administered after the initial dose as needed to maintain an adequate level of anesthesia. Recording was performed as described previously (31). Left ventricular end-systolic and enddiastolic dimensions (LVESD and LVEDD, respectively) were measured from the LV M-mode tracing. Percent fractional shortening (\%FS) of the LV was calculated as \%FS $=($ LVEDD - LVESD $) /$ LVEDD $\times 100$.

In vitro infection assay. Cardiomyocytes were prepared using the Percoll gradient method as described previously (27) and were plated in 24-well plates at a density of $2 \times 10^{5}$ cells per well. After incubation in serum for 24 hours, the cultures were washed and infected with recombinant adenoviruses containing the genes for Myc-tagged SOCS1, SOCS3, or LacZ. Expression of SOCS1 in this construct required Cre recombination of LoxP sites (32); therefore, an adenoviral Cre expression vector was added to all plates. The myocytes were infected for 8 hours with an MOI of 2 viral particles per cell for the cDNA expression vector of interest, and 2 viral particles per cell of the adenovirus Cre vector, for a total of 4 adenoviral particles per cell in all wells, similar to that previously described (32). The cells were washed and cultured in serum-free medium for 24 hours, after which IFN- $\gamma(10 \mathrm{ng} / \mathrm{ml})$, IFN- $\beta(1,000$ $\mathrm{IU} / \mathrm{ml})$, or CT-1 (1 nM) was added to the media for 24 hours. This was followed by CVB3 infection at a MOI of 100 . At 30 hours after CVB3 infection, the cytopathic effect was quantitated by an observer blinded to the experimental conditions who counted the number of the cells with nuclei that were normally stained by hematoxylin staining.

Luciferase assay. CT-1-dependent STAT3 reporter activity was assayed as described previously (18) in cardiomyocytes transfected with AAV shuttle plasmid, 
AAV dnSOCS1 plasmid, and/or pcDNA3-SOCS1. $\mathrm{NF}-\kappa \mathrm{B}$ activity was measured by cotransfection of a NF- $\kappa B-l u c i f e r a s e$ construct that contained six copies of the NF- $\mathrm{KB}$ binding site upstream from luciferase (a generous gift from B. Greenberg and R. Cowling). In all reporter assays, $0.5 \times 10^{6}$ cardiomyocytes plated in six-well dishes were used for transfection with the Lipofectamine PLUS reagent (Invitrogen Corp., San Diego, California, USA).

Direct injection of AAV into the heart. Mice were anesthetized intramuscularly with a mixture of ketamine $(100 \mathrm{mg} / \mathrm{kg})$ and xylazine $(5 \mathrm{mg} / \mathrm{kg})$ followed by incision of the abdominal wall. After elevating the xiphoid process, the vector solutions $\left(10^{11}\right.$ genome copies per milliliter) were injected into the heart through the diaphragm using a $2-\mathrm{ml}$ insulin syringe with a 28 -gauge needle ( $20 \mu \mathrm{l}$ for one injection). After the injection, the skin and peritoneum were closed with a continuous running suture.

Statistical analysis. The results of virus titer, in vitro infection assay, luciferase assay, and echocardiography are expressed as means \pm SE. Statistical significance was evaluated using the unpaired Student's $t$ test for comparisons between two means. Bonferroni correction was performed for multiple comparisons. For the survival rate after CVB3 inoculation, the differences between two groups were analyzed by the log-rank (Mantel-Cox) test. $P$ values less than 0.05 were considered statistically significant.

\section{Results}

Correlation of virus-induced cardiac injury and JAK-STAT activation. To determine whether the JAK-STAT pathway is altered in CVB3-infected hearts, 4-week-old wild-type $\mathrm{Balb} / \mathrm{c}$ mice were intraperitoneally injected with $10^{3}$ PFUs of CVB3. Protein extracts from the heart were analyzed on days 1-3 after infection. We focused on STAT1 and STAT3 as key effectors of IFNand gp130-mediated signaling in the heart. The gp130 signaling is important for cardiac cell survival; howev$\mathrm{er}$, it is not known if it has a role in the pathogenesis of viral infection. On the third day, both STAT1 and STAT3 were strongly activated, as demonstrated by protein phosphorylation (Figure 1a). We also found induction of IFN-responsive genes such as IFN regulatory factor-1 (IRF1) and Fc $\gamma$ RI (Figure 1b). These findings are consistent with activation of IFN and gp130 signaling in the heart at this early stage of infection. Importantly, the intrinsic negative regulators of IFN and gp130 signaling, SOCS1 and SOCS3, were strongly expressed at a time similar to that of the induction of STAT phosphorylation, indicating

\section{Figure 1}

Correlation of CVB3-induced cardiac injury and JAKSTAT activation. (a) Mice were infected with CVB3. Protein lysate from the heart was blotted at the indicated days after CVB3 inoculation and probed with the antibodies indicated. (b) Northern blot of total RNA from the heart after CVB3 inoculation was probed for IRF1, Fc $\gamma R$ I, SOCS1, and SOCS3 expression. 28S and 18S RNA are shown as controls. (c) Virus titer and the disruption of cardiac cell membrane within 5 days after CVB3 inoculation. Four-week-old male Balb/c mice were inoculated with $10^{3}$ PFUs of CVB3 intraperitoneally and sacrificed at the day indicated. Evans blue dye was injected intraperitoneally 4 hours before the sacrifice (4). The left panel shows the time course of virus titer (solid line) and the percentage of Evans blue dye-positive area in the heart (gray bars). The Evans blue dye-positive areas were quantitated using NIH image software $(\mathrm{NIH}$, Bethesda, Maryland, USA). The right panels show Evans blue dye-negative (Day 0) and positive (Day 3 ) sections (the brighter red staining areas). The data were collected from three mice for each time point and expressed as means \pm SE. (d) Dual immunostaining of the infected heart demonstrates that the cells that are positive for Evans blue dye are also positive for viral capsid proteins. The left panel shows immunofluorescent staining with anti-CVB3 antibody (green), and the center panel shows Evans blue dye (red) uptake in the same field. The right panel is a merged image. Scale bars: $1 \mathrm{~mm}$ (c); $50 \mu \mathrm{m}$ (d). P-STAT1, phospho-STAT1; P-STAT3, phospho-STAT3.
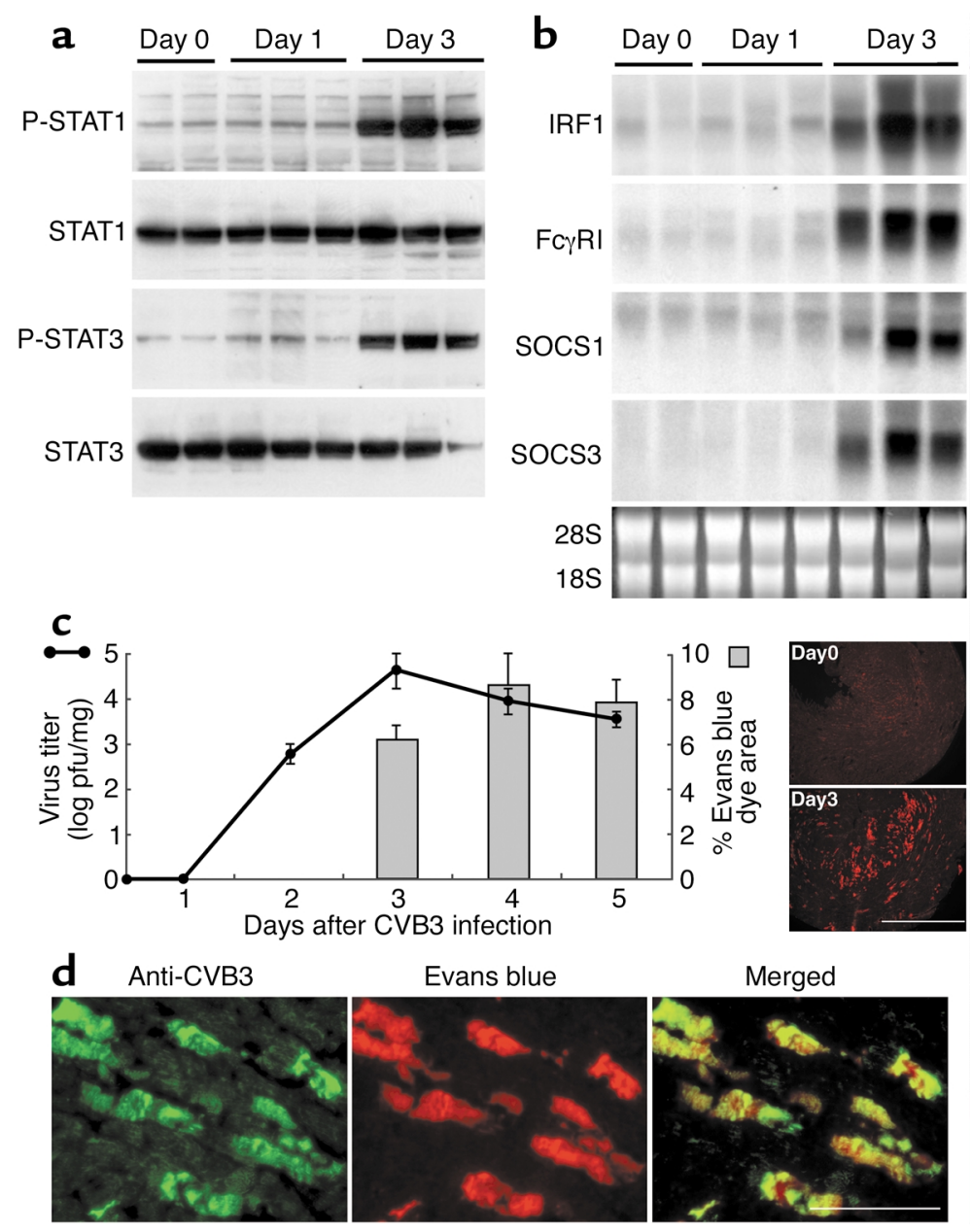
a

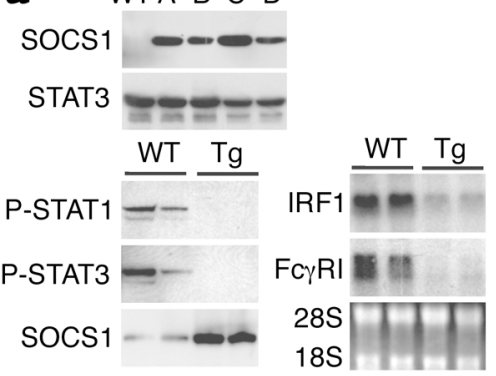

C
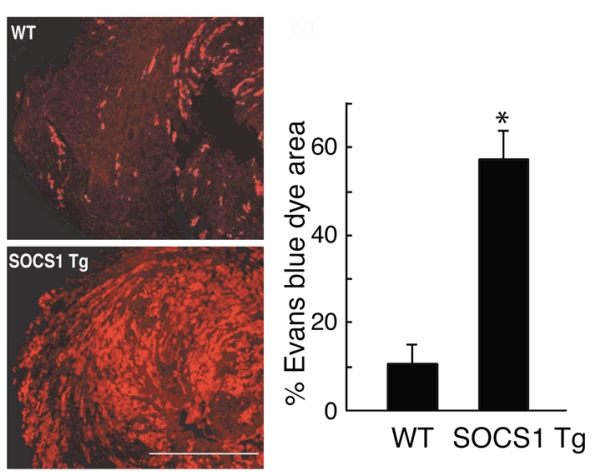

b

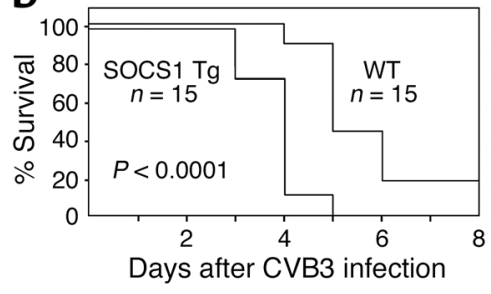

$18 \mathrm{~S}$

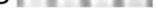

$\mathbf{e}$

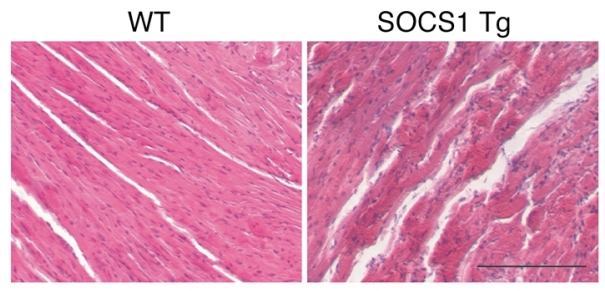

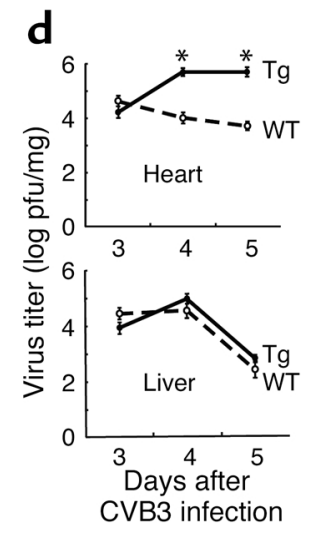

SOCS1 $\mathrm{Tg}$

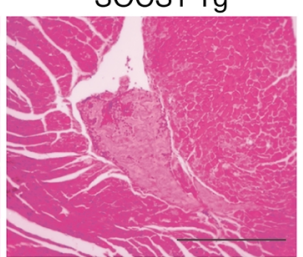

Figure 2

Increased myocardial injury, virus replication, and mortality in SOCS1 transgenic mice. (a) Expression of Myc-tagged SOCS1 in the heart was compared among four SOCS1 transgenic lines (A-D) by immunoblotting with anti-Myc antibody (upper panel). Heart tissue extracts for protein (lower left panel) and RNA (lower right panel) from two lines of wild-type and two lines of transgenic mice (A and $B$ ) were prepared three days after CVB3 inoculation and probed as shown. (b) Wild-type and SOCS1 transgenic mice (4-week-old males) were inoculated with $10^{3}$ PFUs of virus $(n=15)$. The survival rate in infected, SOCS1 transgenic mice was significantly lower than in infected, wild-type littermates $(P<0.0001)$. (c) Evans blue dye uptake in the heart was markedly increased in surviving SOCS1 transgenic mice on day 4 after infection (red stain, left panels). The percent area of Evans blue staining in the hearts is shown (right panel; mean $\left.\pm \mathrm{SE}, n=3,{ }^{*} P<0.01\right)$. (d) Increased viral titer in the heart but not the liver of SOCS1 transgenic mice. Virus titers in SOCS1 transgenic and wildtype hearts and livers from 3-5 days after infection (mean $\pm \mathrm{SE}, n=3,{ }^{*} P<0.01$ comparing SOCS1 transgenic mice with wild-type littermates). (e) Hematoxylin and eosin stains of representative wild-type and SOCS1 transgenic mouse hearts 4 days after infection. Note the thrombus in the center of the ventricle (right panel). Scale bars: $1 \mathrm{~mm}$ (c); $100 \mu \mathrm{m}$ (e, middle); $200 \mu \mathrm{m}$ (e, right). Tg, transgenic; P-STAT1, phospho-STAT1; P-STAT3, phospho-STAT3. activation of the JAK-STAT-SOCS circuit at this early time point (Figure $1 \mathrm{~b}$ ). To determine the correlation between activation of the JAK-STAT-SOCS circuit and viral infection, we measured the virus titer in the heart by a plaque-forming assay. The virus titer began to increase at 2 days and peaked at 3 days after infection (Figure 1c). These results demonstrate that the time point at which JAK-STAT signaling is activated occurs soon after viral infection is detected in the heart, demonstrating the potentially important role of JAKSTAT signaling in the early stages of infection. As shown previously (4), viral infection of the heart was associated with disruption of the sarcolemma that is detected as Evans blue dye staining in the heart. The Evans blue dye colocalized with the presence of virus

\section{Figure 3}

Deterioration of cardiac function after CVB3 inoculation in SOCS1 transgenic mice. Echocardiography was performed 3 days after virus inoculation ( $n=3$ mice per group). Upper panels show that the LVEDD and LVESD were significantly elevated in transgenic mice (black bars) as compared with wild-type (white bars). The \%FS, a parameter of cardiac function, was significantly decreased in transgenic mice as compared with wild-type mice. The lower panel shows the typical M-mode image of the two groups. Results are shown as means \pm SE. ${ }^{*} P<0.01$ for the comparison of SOCS1 transgenic mice with wild-type littermates. $\mathrm{Tg}$, transgenic. in the cell (Figure 1d), indicating that the disrupted sarcolemmal membrane is the direct result of CVB3 infection. We quantitated the percent area of Evans blue dye in the heart section as a marker of virus-mediated cytopathic effect (Figure 1c, gray bars). The disruption of the sarcolemma started at day 3 and peaked at day 4 , demonstrating the importance of this time period in the disease process.
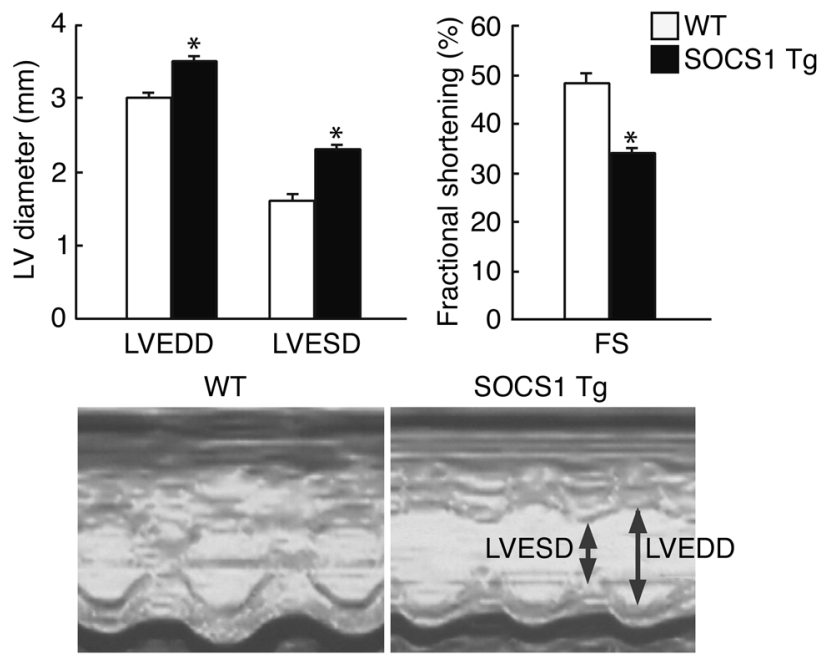
Increased virus replication and myocardial injury in SOCS1 transgenic mice. Since JAK-STAT signaling and its negative regulator, SOCS, are induced in CVB3-infected hearts, we sought to determine the effect of SOCS expression and its potential role as a negative regulator of JAK activation within the infected cardiac myocyte. We therefore generated transgenic mice expressing a Myc-tagged SOCS1 under the control of the cardiac myocyte-specific, $\alpha$-myosin heavy-chain ( $\alpha$-MHC) promoter. Transgene expression was confirmed by immunoblotting with an anti-Myc antibody in four mouse lines (Figure 2a). Pups of SOCS1 transgenic mice were born normally and grew to adulthood without increased mortality. Histological examination of SOCS1 transgenic mice hearts at 16 weeks revealed no evidence of necrosis, ventricular fibrosis, or myofibrillar disarray. Echocardiography also revealed no difference in left ventricular function and wall thickness in SOCS1 transgenic mice when compared with littermate controls (data not shown). Thus, global cardiac structure and function were normal in uninfected SOCS1 transgenic mice.

To determine whether expression of SOCS1 and subsequent inhibition of JAK signaling could have a functionally significant effect in the setting of infection with the cardiotropic CVB3, we inoculated SOCS1 transgenic mice that had been backcrossed into the $\mathrm{Balb} / \mathrm{c}$ strain, which is highly susceptible to CVB3 infection, and their wild-type littermates with CVB3. Consistent with the fact that SOCS1 inhibits JAK signaling stimulated by a variety of cytokines $(13,14,17)$, we found that both STAT1 and STAT3 activation and induction of IFN-responsive genes by CVB3 infection were totally inhibited in the SOCS1 transgenic mice, indicating that SOCS1 transgenic mice may be resistant to stimulation by IFNs and gp130-activating cytokines (Figure 2a). In addition, CVB3-infected SOCS1 transgenic mice had significantly earlier mortality when compared with their wild-type littermates. By day 4 after infection, more than $90 \%$ of SOCS1 transgenic mice were dead. Less than $10 \%$ of the infected controls were dead at the same time point (Figure $2 \mathrm{~b})$. To determine whether the increased mortality in the SOCS1 transgenic mice was associated with increased myocardial injury, we quantitated the Evans blue dye area in SOCS1 transgenic mice and wild-type littermates on day 4 after infection, before the mice had died from the infection. We found that the percent area of myocardial injury in SOCS1 transgenic mice was markedly increased as compared with that of wild-type littermates (Figure 2c). The virus titer in the heart in SOCS1 transgenic mice on days 4 and 5 after CVB3 infection was also much higher when compared with that of wild-type littermates. The viral titer in the liver was not elevated in the transgenic mice (Figure 2d). Hematoxylin and eosin staining of the hearts from SOCS1 transgenic mice and wild-type littermates at 4 days after CVB3 infection demonstrated large areas of necrotic myofibers in SOCS1 transgenic mice, whereas there were only scattered foci of myocyte necrosis in the wild-type littermates. Incidentally, left ventricular mural thrombus was observed only in the SOCS1 transgenic mice, a finding that is likely to be secondary to the extent of myocardial damage (Figure 2e). Occasional mononuclear cells were present in the myocardium, but the extent of mononuclear cell infiltration was similar between SOCS1 transgenic mice and wild-type littermates at this early stage of infection, indicating that increased myocardial injury in SOCS1 transgenic mice is not secondary to increased mononuclear cell infiltration.

Acute cardiomyopathy in SOCS1-transgenic mice. To determine whether SOCS1 expression with its inhibition of JAK signaling in the cardiac myocyte is sufficient to
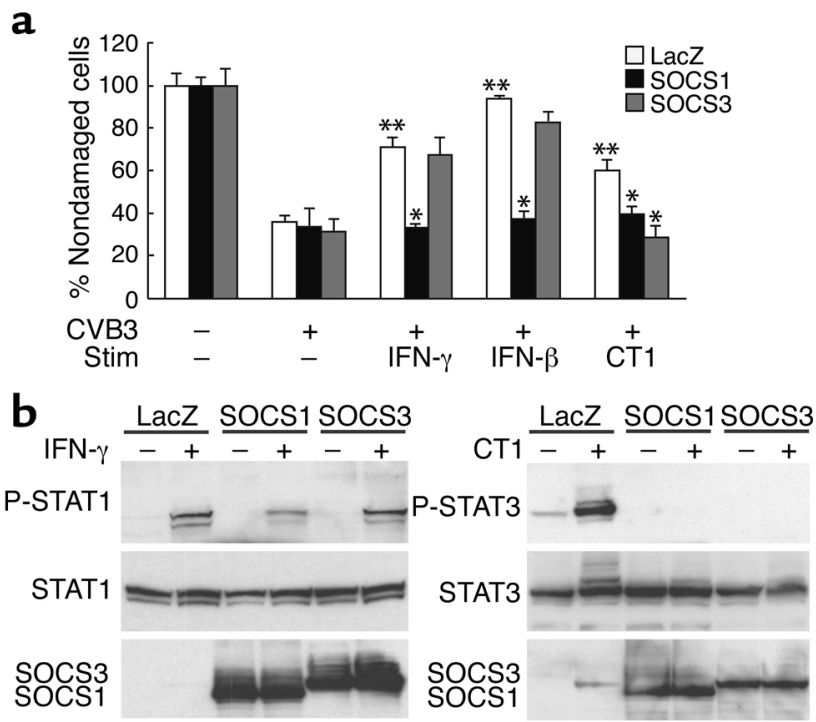

\section{Figure 4}

Effect of SOCS1 and SOCS3 on the cytoprotective effect of IFNs and CT-1 in cultured cardiomyocytes. (a) Myc-tagged SOCS1 or SOCS3 was expressed in neonatal rat cardiomyocytes with the use of recombinant adenovirus vectors (20) (black or gray bars, respectively). The vector containing the LacZ gene was used as a control for adenoviral vector infection (white bars). After transduction with the adenoviral vectors, the cells were stimulated with IFN- $\gamma$, IFN- $\beta$, or CT- 1 for 24 hours. Cells were then infected with CVB3 (+) or maintained without virus (-) for another 30 hours. The number of cells that remained on the plate after CVB3 infection was quantitated and reported as a percentage of cells in the wells not infected with CVB3. The data are from five independent experiments and are expressed as means \pm SE. ${ }^{*} P<0.01$ for the comparison with cells transduced with adenovirus LacZ, stimulated with cytokines, and infected with CVB3. ${ }^{*} P<0.01$ for the comparison with cells transduced with adenovirus LacZ, not stimulated with cytokines, and infected with CVB3. (b) Myocytes were incubated with adenovirus LacZ, adenovirus SOCS1, or adenovirus SOCS3, serum depleted for 24 hours, and then stimulated with $1000 \mathrm{ng} / \mathrm{ml} \mathrm{IFN}-\gamma$ for 5 hours or $1 \mathrm{nM} \mathrm{CT-1}$ for 10 minutes. Total cell extracts were prepared and blotted with phospho-STAT1, STAT1, phospho-STAT3, and STAT3 antibodies. SOCS1 and SOCS3 expression were confirmed with an antiMyc antibody. Representative Western blots from three independent experiments are shown. SOCS1, adenovirus containing Myc-tagged SOCS1 gene; SOCS3, adenovirus containing Myc-tagged SOCS3 gene; LacZ, adenovirus containing LacZ; Stim, stimulated, P-STAT1, phospho-STAT1; P-STAT3, phospho-STAT3. 
a

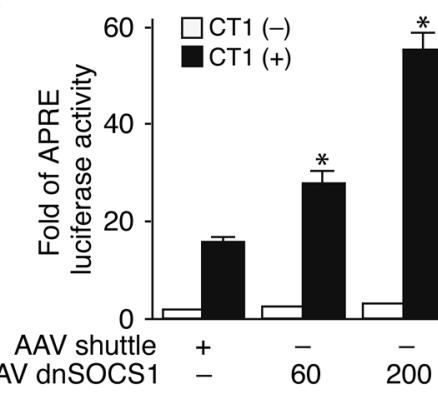

b

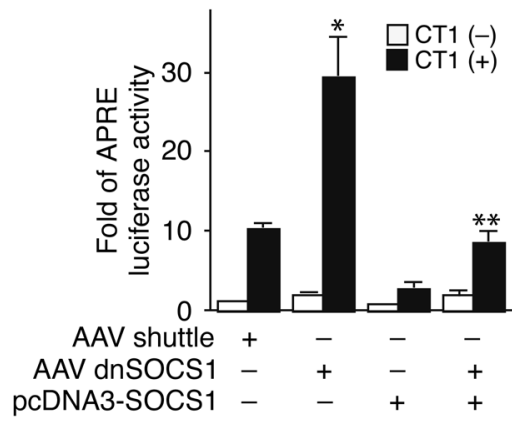

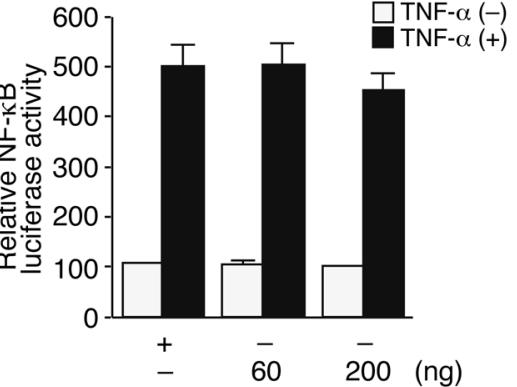

C

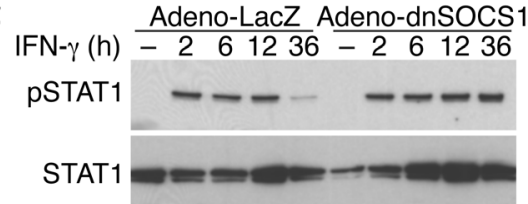

CT1 (h) $-0.51 .5612-0.51 .5612$ PSTAT3

STAT3

dnSOCS1

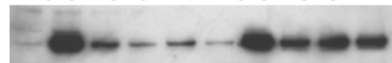

\section{Figure 5}

Augmentation of the JAK/STAT pathway by dnSOCS1 in cardiac myocytes. ( $\mathbf{a}$ and $\mathbf{b}$ ) Cardiomyocytes were transfected with a plasmid mixture containing the APRE-luciferase reporter gene (200 $\mathrm{ng}$ ), the NF-KB-luciferase reporter gene (200 ng), the $\beta$-galactosidase gene $(100 \mathrm{ng})$, the AAV shuttle plasmid, or the indicated concentrations of dnSOCS1 plasmid. After transfection, cells were incubated in the presence or absence of $1 \mathrm{nM} \mathrm{CT-1}$ or $20 \mathrm{ng} / \mathrm{ml} \mathrm{TNF-} \alpha$ for 6 hours, and cell extracts were prepared. Data normalized with the $\beta$-galactosidase activity are shown. The experiments were repeated three times. Results are expressed as means \pm SD. ${ }^{*} P<0.01$ for the comparison of CT- 1 with the AAV shuttle. ${ }^{*} P<0.01$ for the comparison of CT- 1 with pcDNA3-SOCS1. (c) Time course of IFN- $\gamma$-induced STAT1 activation and CT-1-induced STAT3 activation in cardiac myocytes. Myocytes were treated with adenovirus LacZ or adenovirus dnSOCS1, were serum depleted for 24 hours, and were then stimulated with $1000 \mathrm{ng} / \mathrm{ml} \mathrm{IFN}-\gamma$ or $1 \mathrm{nM} \mathrm{CT}-1$ for the indicated period, respectively. Total cell extracts were prepared and blotted with phospho-STAT1, STAT1, phospho-STAT3, and STAT3 antibodies. The dnSOCS1 expression was confirmed with an anti-Myc antibody. Data from one experiment are presented; two additional experiments yielded comparable results. P-STAT1, phospho-STAT1; P-STAT3, phospho-STAT3; APRE, acute-phase response element.

an important innate antiviral defense mechanism, and that inhibition of the JAK-STAT pathway by increased expression of SOCS can have a detrimental effect on the antiviral defense mounted by the infected host cell.

Inhibition of antiviral effect of cytokines by SOCS in cultured myocytes. Given the importance of JAK-STAT signaling in the SOCS1 transgenic mice, we sought to determine whether cytokines that activate JAKSTAT signaling could inhibit the CVB3-mediated cytopathic effect in isolated cardiac myocytes. We found that IFN- $\gamma$, IFN- $\beta$, and CT- 1 , a gp130-activating cytokine, inhibited the virus-mediated cytopathic effect. We also found that expression of SOCS1 using an adenoviral expression vector inhibited the protective effect of both IFNs and CT-1, whereas SOCS3 expression did not have a significant effect on the protective effect of IFNs in this model system but inhibited the protective effect of the gp130 ligand, CT-1 (Figure 4a). These data indicate that expression of SOCS1 is likely to inhibit both IFN receptor and gp130 signaling cascades in cardiac myocytes, both of which could have an important role in limiting the virus-mediated cytopathic effect. Furthermore, this demonstrates that gp130-mediated activation of JAK-STAT may be an important inhibitor of the virus-mediated cytopathic effect and that either SOCS1 or SOCS3 expression may

affect cardiac function with CVB3 infection, echocardiography was performed before and 3 days after CVB3 infection. LV function was normal in both wild-type and SOCS1 transgenic mice before infection. At 3 days after CVB3 infection, LV function and chamber size were near normal in wild-type mice. On the other hand, chamber dilation in the SOCS1 transgenic mice was manifested as a significant increase in LVEDD and LVESD. There was also a significant decrease in the fractional shortening (Figure 3). All of these findings are typical of those seen with acute myocarditis and dilated cardiomyopathy in humans. Thus, cardiac myocyte-specific expression of SOCS1 with its associated inhibition of JAK signaling in myocardial cells resulted in robust virus replication and substantial myocardial injury, leading to acute left ventricular dysfunction and rapid death in mice. This demonstrates that JAK-STAT signaling within the cardiac myocyte is adversely affect the cytopathic limiting potential of cytokines in the heart. Consistent with the result from this virus-mediated cytopathic effect, ectopic SOCS1 expression inhibited both IFN- $\gamma$-induced STAT1 activation and CT-1-induced STAT3 activation, whereas ectopic SOCS3 expression inhibited CT-1-induced STAT3 activation but not IFN- $\gamma$-induced STAT1 activation in cardiomyocytes (Figure $4 \mathrm{~b}$ ).

Augmentation of cytokine-induced JAK-STAT activation by dnSOCS1 in cardiomyocytes. Recently, Hanada et al. demonstrated that dnSOCS1, which has a point mutation (F59D) in a functionally critical kinase inhibitory region of SOCS1, strongly augmented cytokine-dependent JAK-STAT activation both in vivo and in vitro (33). The authors identified the degradation of SOCS1 in thymocytes prepared from transgenic mice that expressed dnSOCS1 in a T cell-specific manner, resulting in the cytokine-induced hyperactivation of JAK and STAT and 

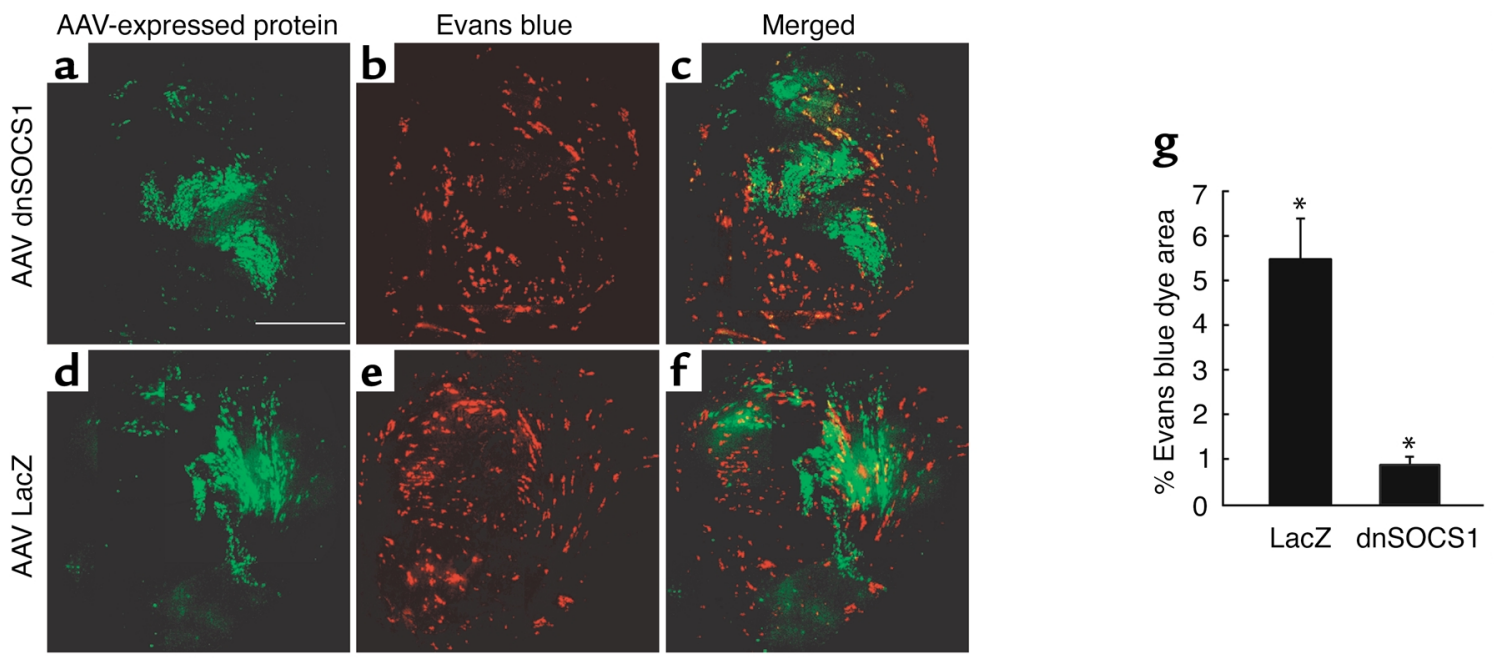

\section{Figure 6}

AAV-directed expression of dnSOCS1 inhibits the CVB3-mediated cytopathic effect. Hearts of mice were directly injected with AAV Myc-tagged dnSOCS1 or AAV LacZ. Two weeks later, the mice were infected with CVB3. Five days after infection, myocardial sections were stained with antiMyc (a) or anti- $\beta$-galactosidase antibodies (d). Evans blue uptake was examined in the same section ( $\mathbf{b}$ and $\mathbf{e})$. The merged images (c and $\mathbf{f})$ show that in the areas where there is expression of dnSOCS1, there is a lack of membrane disruption due to the virus infection, as compared with areas of the myocardium that express LacZ. The percent area of Evans blue dye staining in the regions of the myocardium that stained positive for dnSOCS1 or LacZ was quantitated from three mice per group $(\mathbf{g})$ and was significantly decreased in dnSOCS1-expressing areas as compared with LacZ-expressing areas. Results are shown as means \pm SE. ${ }^{*} P<0.01$ for the comparison with LacZ-transduced areas. Scale bars: $1 \mathrm{~mm}(\mathbf{a}-\mathbf{f})$.

hyperproliferation of $\mathrm{T}$ cells. To define the dnSOCS1 function in cardiomyocytes, a STAT3 reporter assay was performed. The AAV-dnSOCS1 plasmid markedly enhanced the CT-1-induced STAT3 activity as compared with AAV shuttle plasmid (Figure 5a). The AAVdnSOCS1 plasmid did not affect tumor necrosis factor- $\alpha$-dependent NF- $\kappa B$ activation. Importantly, AAV-dnSOCS1 plasmid significantly overcame the inhibition of CT-1-induced STAT3 activation by SOCS1 (Figure 5b). Next, we examined the effect of dnSOCS1 on the IFN- $\gamma$-induced STAT1 phosphorylation and the CT-1-induced STAT3 phosphorylation using this adenovirus of dnSOCS1 and cardiomyocytes. As shown in Figure $5 c$, the phosphorylation of STATs in cardiomyocytes expressing dnSOCS1 was sustained longer than that in cardiomyocytes expressing LacZ. These data indicate that ectopic expression of dnSOCS1 in cardiomyocytes enhances responses to cytokines through the SOCS1 inhibition.

Inbibition of virus-induced cardiac injury by inbibition of SOCS. Since CVB3 infection induces both SOCS1 and SOCS3, it is possible that if SOCS1 and SOCS3 could be inhibited in the heart that activation of JAK-STAT signaling by endogenous cytokines might be able to inhibit viral replication more effectively. To test this hypothesis, we generated an AAV vector to express a Myc-tagged dnSOCS1. The dnSOCS1 destabilizes both endogenous SOCS1 and SOCS3 and enhances JAKSTAT signaling (33). This augments the JAK-STAT signaling that occurs with either IFNs or gp130 receptor stimulation. The AAV vector expressing either the dnSOCS1 or, as a control, LacZ, was injected directly into the heart. Two weeks after the gene transfer, the mice were inoculated with CVB3. The extent of myocardial injury was examined by Evans blue dye uptake 5 days after infection (since the mice were 2 weeks older than those studied previously, their rate of mortality was lower). Expression of dnSOCS1 and LacZ was determined by immunostaining with antiMyc or anti- $\beta$-galactosidase antibodies, respectively. In the areas of the myocardium that expressed dnSOCS1, there was almost no Evans blue dye staining. (Figure 6, $a-c$ ), whereas Evans blue dye staining in the area of the myocardium that expressed LacZ was not different from areas not transduced with the AAV vectors (Figure 6, d-f). A quantitative evaluation of three separate sections in each of three mice from each group showed a significant difference between the two groups (Figure 6g). As demonstrated previously (Figure 1d), the Evans blue dye staining colocalized with viral infection, and there was no significant evidence of CVB3 infection in the area of the myocardium that expressed the dnSOCS1 (data not shown). Thus, inhibition of SOCS in the myocardium effectively prevented the CVB3-induced acute myocardial injury and inhibited viral replication. These findings demonstrate that strategies aimed at inhibition of SOCS could potentiate the innate antiviral actions of cytokines that stimulate JAK-STAT activation.

\section{Discussion}

We have focused on the role of SOCS as negative-feedback regulators of JAK signaling and their role in the innate host defense within the cardiac myocyte against viral infection. We have demonstrated that JAK-STAT signaling is activated in the heart of infected mice and that 

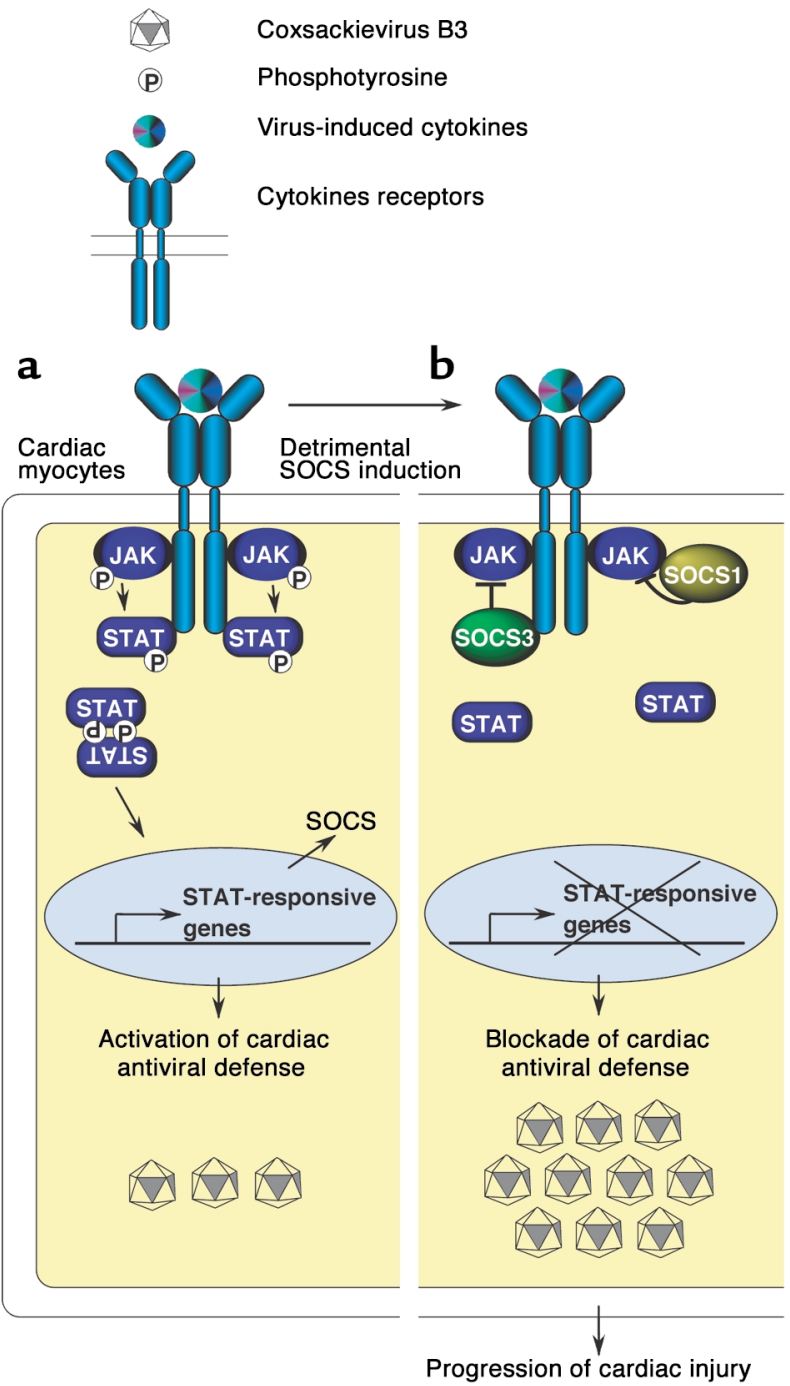

it is required for the early innate defense against enteroviral infection in the heart. Although it is clear that the cellular immune response is an important defense against viral infection with cardiotropic viruses $(5,34)$, these results demonstrate the importance of the innate antiviral defense system within the cardiac myocyte. Since JAKSTAT activation occurs before there is significant infiltration with mononuclear lymphocytes, it appears to have a key role in the control of viral replication at the early stages of viral infection (Figure 7a).

Viral infection induces expression of cytokines that activate JAK signaling - such as IFN- $\alpha / \beta$, IFN- $\gamma$, gp130-related cytokines (CT-1, IL-6), IL-10, and IL-12 - at the early stages of myocarditis (35-39). The exogenous administration of these cytokines has been shown to ameliorate the severity of viral myocarditis in mice. However, these protective effects are not complete. We recently reported that whole-animal knockout of the IFN- $\alpha / \beta$ receptor had no significant effect on the early stages of viral replication in the heart. Disruption of the IFN- $\gamma$ receptor had only a very small effect on early viral replication in the heart (23). Thus,

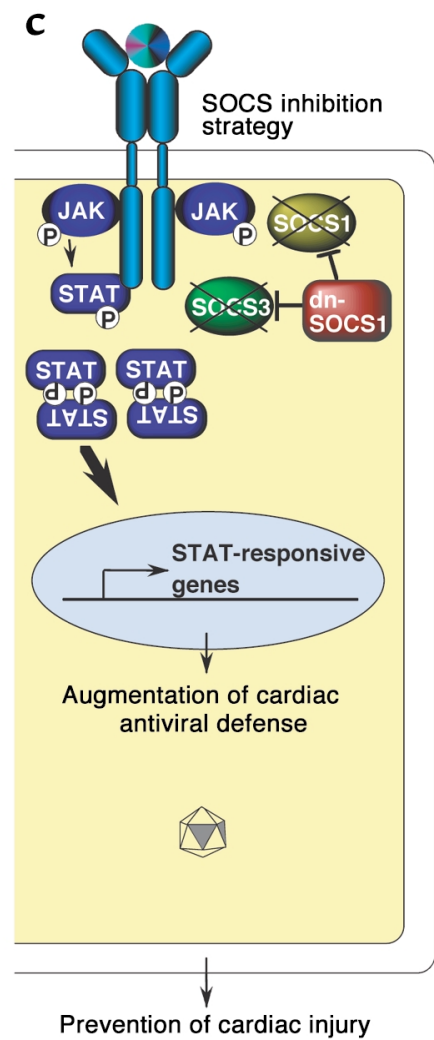

Figure 7

JAK-STAT antiviral defense in the cardiac myocyte and SOCS inhibition to limit early virusinduced cardiac injury. (a) Virus infection of the heart stimulates cytokine-receptor signaling through the JAK-STAT pathway. Activation of the JAKSTAT pathway induces antiviral target gene transcription stimulating the innate viral defense in the cardiac myocyte. (b) While activation of the JAKSTAT pathway has an important role in antiviral defense, phosphorylated STAT also induces SOCS expression that attenuates the innate antiviral defense by inhibiting JAK signaling. As in SOCS1-transgenic mice, increased expression of SOCS in cardiac myocytes results in robust virus replication and cardiac injury. (c) The strategies aimed at inhibition of SOCS potentiate the innate antiviral actions of cytokines that utilize the JAK-STAT pathway, resulting in the prevention of virus-mediated myocardial injury. P, phosphorylated. it appears that administration of a single cytokine or knockout of a single cytokine receptor does not have a profound effect on the early stages of viral myocarditis. On the other hand, inhibition of JAKSTAT signaling by SOCS has a marked effect on viral replication and cardiac injury, suggesting that stimulation of JAK-STAT signaling by multiple cytokines such as IFN- $\alpha / \beta$, IFN- $\gamma$, and/or gp130-related cytokines may be necessary for full stimulation of the potent innate defense against viral infection of the cardiac myocyte in the intact heart.

The common receptor of the IL- 6 family of cytokines, gp130, has been demonstrated to play an important role in cardiac myocyte cell survival $(40,41)$. Little is known, however, about the effect of gp130 signaling on the virus-induced cell damage. In this study, we found that STAT3, the main downstream molecule of gp130 signaling, is activated at the early stages of myocarditis (Figure 1a) and that CT-1 prevents the cardiac myocyte cell damage that occurs with CVB3 infection in vitro (Figure 4a). We also found that another gp130-interacting cytokine, IL-6, inhibits cardiac 
myocyte cell damage from CVB3 infection in vitro (data not shown). Using the experimental approach involving adenoviral infection coupled with CVB3 infection, one cannot entirely exclude the possibility that some of the observed effects could be due to unanticipated effects of infection with two viruses. However, the overall results indicate that gp130 signaling in the myocyte could have a role in the pathogenesis of the early stage of myocarditis. We have previously shown that disruption of the dystrophinglycoprotein complex has a role in the pathogenesis of viral myocarditis. Since stimulation of gp130 signaling stimulates a hypertrophic phenotype with changes in cell shape (42), it will be interesting to determine whether there are alterations in cytoskeletal proteins after gp130 stimulation that are involved in the cytoprotective effect that is associated with activation of JAK-STAT signaling in the infected cardiac myocyte. Infection of the cardiac-specific gp130 knockout mice with CVB3 will allow direct evaluation of the role of the cardiac gp130 signaling pathway in the pathogenesis of the early stages of myocarditis.

The balance between JAK-STAT activation and SOCS expression has important effects in normal and infected tissues. For example, knockout of SOCS1 leads to a lethal phenotype by $2-3$ weeks of age that is associated with fatty degeneration and necrosis in the liver (43). The detrimental effect of SOCS1 deficiency can be ameliorated by inhibiting IFN- $\gamma$ either with antibodies to IFN- $\gamma$ or by breeding the SOCS1 knockout mice with IFN- $\gamma$-deficient mice (16). These studies highlighted the importance of SOCS1 as a key regulator of IFN- $\gamma$ signaling in the uninfected mouse. Increases in antiviral cytokines such as IFN during viral infection are important for limiting replication of the virus and controlling the extent of damage in certain tissues, as has been demonstrated in the liver and pancreas $(23,44)$. However, our data demonstrate that with enteroviral infection of the heart, the upregulation of SOCS1 expression has a maladaptive effect in the early stages of viral replication and facilitates replication of the virus by preventing the full action of the JAK-STAT signaling pathway (Figure $7 \mathrm{~b}$ ). This may be due to inhibition of IFN and gp130 signaling. The induction of SOCS1 and SOCS3 on day 3 after infection may explain the fact that IFN administration is only beneficial in CVB3-induced myocarditis when given early (22). Our data do not exclude the possibility that SOCS expression may be beneficial in late stages of infection or in other disease states that activate JAK-STAT signaling in the heart. Inhibition of SOCS at later time points after infection with CVB3 could be tested using inducible expression of dnSOCS1.

Since inhibition of SOCS potentiates the antiviral effects of JAK-STAT signaling during the early stages of viral infection, small-molecule antagonists of SOCS or tissue-specific vector delivery of SOCS inhibitors during the stages of viral infection in which there is active viral replication may prove to be a clinically valuable strategy to enhance the protective effect of the antiviral cytokines that operate through JAK-STAT signaling (Figure 7c). The strategy of SOCS inhibition for treatment of early virus-mediated organ damage might be also useful for other viral diseases or cancers such as chronic viral hepatitis, chronic myeloid leukemia, and renal cell carcinoma. Although it has been shown that IFN therapy is effective for these conditions, some patients are resistant to IFN therapy $(45,46)$. Sakamoto et al. reported that SOCS1 and SOCS3 are highly expressed without cytokine stimulation and that cytokine-induced JAK-STAT activation is markedly reduced in IFN-resistant leukemia cell lines, suggesting that reduced activation of JAK by aberrant SOCS induction could be a mechanism of IFN resistance (47). The strategy of SOCS inhibition may be effective not only for early virus-induced organ damage, including myocarditis, but also for patients who are resistant to cytokines such as IFN.

\section{Acknowledgments}

We would like to thank Jude Samulski for providing necessary AAV constructs; Janelle Stricker, Chisa Suzuki, Julie Anderson, and Kim Weldy for expert assistance; and Andrea Dorner and Antine Stenbit for helpful discussions and reagents. This research was supported by the Jean LeDucq Foundation. H. Yasukawa was supported by a Banyu Fellowship Award in Cardiovascular Medicine sponsored by Banyu Pharmaceutical Co. and The Merck Company Foundation. H. Duplain was supported by the Botnar Center for Clinical Research, the Roche Research Foundation, and the Novartis Foundation.

1. Rose, N.R. 2000. Viral damage or "molecular mimicry" - placing the blame in myocarditis. Nat. Med. 6:631-632.

2. Liu, P., Martino, T., Opavsky, M.A., and Penninger, J. 1996. Viral myocarditis: balance between viral infection and immune response. Can. J. Cardiol. 12:935-943.

3. Baboonian, C., Davies, M.J., Booth, J.C., and McKenna, W.J. 1997. Coxsackie B viruses and human heart disease. Curr. Top. Microbiol. Immunol. 223:31-52.

4. Badorff, C., et al. 1999. Enteroviral protease 2A cleaves dystrophin: evidence of cytoskeletal disruption in an acquired cardiomyopathy. Nat. Med. 5:320-326.

5. Knowlton, K.U., and Badorff, C. 1999. The immune system in viral myocarditis: maintaining the balance. Circ. Res. 85:559-561.

6. Liu, P., et al. 2000. The tyrosine kinase p56lck is essential in coxsackievirus B3-mediated heart disease. Nat. Med. 6:429-434.

7. Muller, U., et al. 1994. Functional role of type I and type II interferons in antiviral defense. Science. 264:1918-1921.

8. Sen, G.C. 2001. Viruses and interferons. Annu. Rev. Microbiol. 55:255-281.

9. Ihle, J.N. 1995. Cytokine receptor signalling. Nature. 377:591-594.

10. Darnell, J.E., Jr. 1997. STATs and gene regulation. Science. 277:1630-1635.

11. Leonard, W.J., and O'Shea, J.J. 1998. Jaks and STATs: biological implications. Annu. Rev. Immunol. 16:293-322.

12. O'Shea, J.J., Gadina, M., and Schreiber, R.D. 2002. Cytokine signaling in 2002: new surprises in the Jak/Stat pathway. Cell. 109:S121-S131.

13. Starr, R., et al. 1997. A family of cytokine-inducible inhibitors of signalling. Nature. 387:917-921.

14. Naka, T., et al. 1997. Structure and function of a new STAT-induced STAT inhibitor. Nature. 387:924-929.

15. Endo, T.A., et al. 1997. A new protein containing an $\mathrm{SH} 2$ domain that inhibits JAK kinases. Nature. 387:921-924.

16. Alexander, W.S., et al. 1999. SOCS1 is a critical inhibitor of interferon gamma signaling and prevents the potentially fatal neonatal actions of this cytokine. Cell. 98:597-608.

17. Yasukawa, H., Sasaki, A., and Yoshimura, A. 2000. Negative regulation of cytokine signaling pathways. Annu. Rev. Immunol. 18:143-164. 
18. Yasukawa, H., et al. 1999. The JAK-binding protein JAB inhibits Janus tyrosine kinase activity through binding in the activation loop. EMBOJ. 18:1309-1320.

19. Nicholson, S.E., et al. 2000. Suppressor of cytokine signaling-3 preferentially binds to the SHP-2-binding site on the shared cytokine receptor subunit gp130. Proc. Natl. Acad. Sci. USA. 97:6493-6498.

20. Yasukawa, H., et al. 2001. Suppressor of cytokine signaling-3 is a biomechanical stress-inducible gene that suppresses gp130-mediated cardiac myocyte hypertrophy and survival pathways. J. Clin. Invest. 108:1459-1467. doi:10.1172/JCI200113939.

21. Auernhammer, C.J., and Melmed, S. 2001. The central role of SOCS-3 in integrating the neuro-immunoendocrine interface. J. Clin. Invest. 108:1735-1740. doi:10.1172/JCI200114662.

22. Lutton, C.W., and Gauntt, C.J. 1985. Ameliorating effect of IFN-beta and anti-IFN-beta on coxsackievirus B3-induced myocarditis in mice. J. Interferon Res. 5:137-146.

23. Wessely, R., Klingel, K., Knowlton, K.U., and Kandolf, R. 2001. Cardioselective infection with coxsackievirus B3 requires intact type I interferon signaling: implications for mortality and early viral replication. Circulation. 103:756-761.

24. Knowlton, K.U., Jeon, E.S., Berkley, N., Wessely, R., and Huber, S. 1996. A mutation in the puff region of VP2 attenuates the myocarditic phenotype of an infectious cDNA of the Woodruff variant of coxsackievirus B3. J. Virol. 70:7811-7818.

25. Kishimoto, C., Kitazawa, M., Hiraoka, Y., and Takada, H. 1997. Extracellular matrix remodeling in coxsackievirus B3 myocarditis. Clin. Immunol. Immunopathol. 85:47-55.

26. Hanakawa, Y., Amagai, M., Shirakata, Y., Sayama, K., and Hashimoto, K. 2000. Different effects of dominant negative mutants of desmocollin and desmoglein on the cell-cell adhesion of keratinocytes. J. Cell Sci. 113:1803-1811.

27. Wang, Y., et al. 1998. Cardiac muscle cell hypertrophy and apoptosis induced by distinct members of the p38 mitogen-activated protein kinase family. J. Biol. Chem. 273:2161-2168.

28. Samulski, R.J., Chang, L.S., and Shenk, T. 1989. Helper-free stocks of recombinant adeno-associated viruses: normal integration does not require viral gene expression. J. Virol. 63:3822-3828.

29. Auricchio, A., Hildinger, M., O'Connor, E., Gao, G.P., and Wilson, J.M. 2001. Isolation of highly infectious and pure adeno-associated virus type 2 vectors with a single-step gravity-flow column. Hum. Gene Ther. 12:71-76

30. Henke, A., Huber, S., Stelzner, A., and Whitton, J.L. 1995. The role of CD8+ T lymphocytes in coxsackievirus B3-induced myocarditis. J. Virol. 69:6720-6728.

31. Tanaka, N., et al. 1996. Transthoracic echocardiography in models of cardiac disease in the mouse. Circulation. 94:1109-1117.

32. Kanegae, Y., et al. 1995. Efficient gene activation in mammalian cells by using recombinant adenovirus expressing site-specific Cre recombinase. Nucleic Acids Res. 23:3816-3821.

33. Hanada, T., et al. 2001. A mutant form of JAB/SOCS1 augments the cytokine-induced JAK/STAT pathway by accelerating degradation of wild-type JAB/CIS family proteins through the SOCS-box. J. Biol. Chem. 276:40746-40754.

34. Cook, D.N., et al. 1995. Requirement of MIP-1 alpha for an inflammatory response to viral infection. Science. 269:1583-1585.

35. Tanaka, T., et al. 2001. Overexpression of interleukin- 6 aggravates viral myocarditis: impaired increase in tumor necrosis factor-alpha. J. Mol. Cell. Cardiol. 33:1627-1635.

36. Kanda, T., et al. 1996. Modification of viral myocarditis in mice by interleukin-6. Circ. Res. 78:848-856.

37. Shioi, T., et al. 1997. Protective role of interleukin-12 in viral myocarditis. J. Mol. Cell. Cardiol. 29:2327-2334.

38. Schmidtke, M., et al. 2000. Cytokine profiles in heart, spleen, and thymus during the acute stage of experimental coxsackievirus B3-induced chronic myocarditis. J. Med. Virol. 61:518-526.

39. Okuno, M., et al. 2000. Expressional patterns of cytokines in a murine model of acute myocarditis: early expression of cardiotrophin-1. Lab. Invest. 80:433-440

40. Sheng, Z., et al. 1997. Cardiotrophin 1 (CT-1) inhibition of cardiac myocyte apoptosis via a mitogen-activated protein kinase-dependent pathway. Divergence from downstream CT-1 signals for myocardial cell hypertrophy. J. Biol. Chem. 272:5783-5791.

41. Hirota, H., et al. 1999. Loss of a gp130 cardiac muscle cell survival pathway is a critical event in the onset of heart failure during biomechanical stress. Cell. 97:189-198.

42. Wollert, K.C., et al. 1996. Cardiotrophin-1 activates a distinct form of cardiac muscle cell hypertrophy. Assembly of sarcomeric units in series VIA gp130/leukemia inhibitory factor receptor-dependent pathways. J. Biol. Chem. 271:9535-9545.

43. Marine, J.C., et al. 1999. SOCS1 deficiency causes a lymphocyte-dependent perinatal lethality. Cell. 98:609-616.

44. Flodstrom, M., et al. 2002. Target cell defense prevents the development of diabetes after viral infection. Nat. Immunol. 3:373-382.

45. Gretch, D. 2001. Mechanism of interferon resistance in hepatitis C. Lancet. 358:1662-1664.

46. Brinckmann, A., et al. 2002. Interferon-alpha resistance in renal carcinoma cells is associated with defective induction of signal transducer and activator of transcription 1 which can be restored by a supernatant of phorbol 12-myristate 13-acetate stimulated peripheral blood mononuclear cells. Br. J. Cancer. 86:449-455.

47. Sakamoto, H., et al. 1998. A Janus kinase inhibitor, JAB, is an interferongamma-inducible gene and confers resistance to interferons. Blood. 92:1668-1676. 